\title{
Clinicopathologic correlation of endometrial stromal sarcomas: a retrospective study of 42 cases
}

\author{
Fengjie Wang ${ }^{1,2}$, Yongfen $\mathrm{Yi}^{1}$, Yi Luo ${ }^{3}$, Jing Chen ${ }^{1}$ \\ ${ }^{1}$ Department of Pathology, Molecular Medicine and Tumor Center, Chongqing Medical University, Chongqing 400016, China; ${ }^{2}$ Science and \\ Technology College of Hubei University for Nationalities, Enshi 445000, China; ${ }^{3}$ Department of Gynecology and Obstetrics, The First Affiliated \\ Hospital of Chongqing Medical University, Chongqing 400016, China \\ Contributions: (I) Conception and design: F Wang, Y Luo; (II) Administrative support: Y Yi; (III) Provision of study materials or patients: All authors; \\ (IV) Collection and assembly of data: J Chen, F Wang, Y Luo; (V) Data analysis and interpretation: J Chen, F Wang; (VI) Manuscript writing: All \\ authors; (VII) Final approval of manuscript: All authors. \\ Correspondence to: Yi Luo. Department of Gynecology and Obstetrics, The First Affiliated Hospital of Chongqing Medical University, Chongqing \\ 400016, China. Email: smileyi@163.com.
}

\begin{abstract}
Background: To evaluate clinicopathologic features and molecular markers associated with prognosis and diagnosis of endometrial stromal sarcomas (ESS) and analyze prognostic values.

Methods: We analyzed the clinic and pathologic records of 42 patients who were diagnosed with ESS from the First Affiliated Hospital of Chongqing Medical University between January 2012 and August 2017 retrospectively, and further explored the correlation between pathological features and prognosis.

Results: The age of the patients ranged from 20 to 74 years (mean: 45.5 years). There were 24 low-grade ESS (LGESS), 16 high-grade ESS (HGESS) and 2 undifferentiated uterine sarcoma (UUS) respectively, of which included 6 recurrent cases involving extra-uterine organs. The tumors of most patients usually showed positive for CD10, Vimentin, estrogen receptor (ER), progesterone receptor (PR), CyclinD1, and negative for SMA and S-100 with immunohistochemical staining. Tumor classification, depth of invasion, and tumor stage showed correlation with the recurrence rate $(\mathrm{P}<0.01,0.05$, and 0.01$)$. CyclinD1 expression was significantly associated with tumor recurrence $(\mathrm{P}<0.01)$, and $\mathrm{ER}, \mathrm{PR}$ with tumor classification $(\mathrm{P}<0.05,0.01)$. Conclusions: Our results demonstrate that CyclinD1 positivity may contribute to tumor recurrence and malignancy associated with ESS. Tumor classification, depth of invasion, and tumor stage show significant association with the recurrences.
\end{abstract}

Keywords: Endometrial stromal sarcoma (ESS); clinicopathologic correlation

Submitted Feb 24, 2018. Accepted for publication May 29, 2018.

doi: $10.21037 /$ tcr.2018.06.12

View this article at: http://dx.doi.org/10.21037/tcr.2018.06.12

\section{Introduction}

Endometrial stromal sarcoma (ESS) is a rare mesenchymal tumor, which accounts for less than $1 \%$ of all uterine malignancies, but it is the second most common uterine malignant mesenchymal tumor, and divided into lowgrade ESS (LGESS), high-grade ESS (HGESS) and undifferentiated uterine sarcoma (UUS) according to the WHO Classification of Tumors of Female Reproductive Organs [2014] (1). LGESS is typically composed of cytologically bland fusiform cells resembling stromal cells of proliferative-phase endometrium, permeating the myometrium as well as the intramyometrial or parametrial vessels $(2,3)$. Both HGESS and UUS often exhibit myometrial invasion, hemorrhage and necrosis, as well as marked nuclear pleomorphism with high mitotic activity $(2,3)$. And UUS is a highly malignant sarcoma, which lacks endometrial stromal differentiation and exhibits a complex karyotype with no specific translocation (1).

Factors such as mitotic index, tumor size, clinical stage, 
pathological grade, positive surgical margins, menopause and age have been reported as potential prognostic parameters, but their use in ESS remains controversial $(4,5)$. A retrospective study (6) reported that CD10/ vimentin were expressed more frequently and CD10negative expression may contribute to the malignant characteristics and recurrence associated with ESS. LGESS characteristically showed diffuse positivity for CD10, estrogen receptor (ER), and progesterone receptor (PR), while HGESS typically showed absent or only focal and weak staining for CD10, ER and PR, diffuse positivity for CyclinD1 as described by one study (7). LGESS usually has an indolent course, whereas HGESS and UUS a more aggressive manner with a poorer prognosis, so it is necessary to distinguish low- and high-grade tumors for pathologists and gynecologists. Although immunohistochemistry (IHC) often plays an adjunct role in the differential diagnosis of different uterine mesenchymal lesions, sensitive and specific IHC markers have not been identified for diagnosis in ESSs (8). How to differentiate and diagnose ESSs correctively remains a difficulty for pathologists and gynecologists, so it is crucial to seek better diagnostic markers and standard surgical treatment to decrease recurrence in clinic.

In this study we analyzed the possibly useful molecular markers that correlate with prognosis and the clinicopathological features of ESSs according to the IHC results retrospectively, of all patients from the First Affiliated Hospital of Chongqing Medical University between January 2012 and August 2017, including 24 LGESS, 16 HGESS, and 2 UUS, in order to provide strategy for clinic practice.

\section{Methods}

Immunohistochemical staining was performed using the avidin-biotin immunoperoxidase method as previously described (8). IHC results were confirmed again, and all pathological slides were reviewed and confirmed by two independent pathologists in terms of the degree of immunoreactivity and the intensity. The degree was scored semi quantitatively on the basis of the percentage of positive cells: negative [0]; minimal positive $(<5 \%)$; focal positive (5-39\%); multifocal positive (40-75\%); and diffuse positive $(>75 \%)$. And intensity of staining (negative, 0 ; weak, $1+$; moderate, $2+$; and strong, 3+). Cytoplasm or nuclear staining showed yellow or yellowish brown as positive cases.

We searched the clinic and pathology files from the First Affiliated Hospital of Chongqing Medical University to identify patients diagnosed with ESS between January 2012 and August 2017. Clinical features included patient's age, tumor size, stage, distant metastasis, surgical operation, clinical outcome, recurrence patterns, and follow-up data that were collected from the medical records. Pathology reports were reviewed to determine the sites of the tumors and the extent of surgical staging.

The International Federation of Gynecology and Obstetrics (FIGO) stage for endometrial carcinoma was adapted for ESS (stage I, tumors limited to the uterus; stage II, involvement of the cervix; stage III, involvement of the pelvis; and stage IV, disease outside the pelvis).

The study was reviewed and approved by the ethics committee of the First Affiliated Hospital of Chongqing Medical University.

Statistical analysis: all statistical analyses were carried out using SPSS 20.0 (SPSS Inc.); two-side Fisher's exact test was used to compare two groups. $\mathrm{P}<0.05$ was considered statistically significant.

\section{Results}

\section{Clinical features}

The clinical features of the patients are summarized in Table 1. The age of the 42 patients ranged from 20 to 74 years (median: 45.5 years). The most common initial clinical presentation included uterine bleeding (17/42, 40.48\%), pelvic pain $(11 / 42,26.19 \%)$ and asymptomatic $(14 / 42$, $33.33 \%)$. Twenty six point nineteen percent $(26.19 \%)$, $54.76 \%$, and $19.05 \%$ patients underwent operation by TAH (total abdominal hysterectomy), TAH + BSO (bilateral salpingo-oophorectomy), and TAH + BSO + PL (pelvic lymphadenectomy) respectively, thus TAH + BSO was the mainstay of surgery. Of all patients, 19 had stage I \& II disease, and 16 had stage III \& IV disease according to the 2009 FIGO stage.

Twenty-nine patients were alive with or free of disease including 10 HGESS and 19 LGESS, 2 died, and others missing in a short-term (2-60 months) follow-up visit. Among all cases, 21.43\% (9/42) developed recurrence after operation within half a year, of which six cases occurred in extra-uterine sites.

\section{Pathologic features}

Table 2 showed the pathological characteristics. According to the criteria of WHO Classification [2014] there were 
Table 1 Clinical features of the 42 patients with ESS

\begin{tabular}{|c|c|}
\hline Parameter & Number (\%) \\
\hline Age, median (years) & 45.5 \\
\hline \multicolumn{2}{|c|}{ Initial clinical presentation } \\
\hline Bleeding & $17 / 42(40.48)$ \\
\hline Pelvic pain & 11/42 (26.19) \\
\hline Asymptomatic & 14/42 (33.33) \\
\hline \multicolumn{2}{|l|}{ FIGO stage } \\
\hline$I+I I$ & $19 / 42(45.24)$ \\
\hline $\mathrm{III}+\mathrm{IV}$ & $16 / 42(38.10)$ \\
\hline Unknown & $7 / 42(16.67)$ \\
\hline \multicolumn{2}{|l|}{ Relapse } \\
\hline Yes & $9 / 42(21.43)$ \\
\hline No & $21 / 42(50.00)$ \\
\hline Unknown & $12 / 42(28.57)$ \\
\hline \multicolumn{2}{|l|}{ Clinical outcomes } \\
\hline Alive & 29/42 (69.05) \\
\hline Dead & $2 / 42(4.76)$ \\
\hline Missing & 11/42 (26.19) \\
\hline \multicolumn{2}{|l|}{ Surgical operation } \\
\hline $\mathrm{TAH}$ & $11 / 42(26.19)$ \\
\hline $\mathrm{TAH}+\mathrm{BSO}$ & $23 / 42(54.76)$ \\
\hline $\mathrm{TAH}+\mathrm{BSO}+\mathrm{PL}$ & 8/42 (19.05) \\
\hline
\end{tabular}

ESS, endometrial stromal sarcoma; TAH, total abdominal hysterectomy; BSO, bilateral salpingo-oophorectomy; PL, pelvic lymphadenectomy; FIGO, Federation of Gynecology and Obstetrics.

24 LGESS, 16 HGESS and 2 UUS confirmed by two independent pathologists. The median diameter of tumor was $4.6 \mathrm{~cm}$. Depth of myometrial invasion $(\leq 1 / 2$ myometrium) and lymph-vascular space involvement (LVSI) were identified in $25(59.52 \%)$ and $8(19.05 \%)$ patients, respectively.

More than 20 IHC markers were used to distinguish different ESSs from other malignancies, here we listed 10 common markers which were meaningful for pathology diagnostics in Table 3. During IHC staining, the tumors of most patients usually showed positivity for CD10, Vimentin, ER, PR, and CyclinD1, and negative for SMA and S-100 as shown in Table 3. Ki67 indexes more than 5\%
Table 2 Pathologic features of the 42 patients with ESS

\begin{tabular}{lc}
\hline Parameter & Number (\%) \\
\hline Tumor size, median $(\mathrm{cm})$ & 4.6 \\
Classification & $24 / 42(57.14)$ \\
LG & $16 / 42(38.10)$ \\
HG & $2 / 42(4.76)$ \\
UUS & \\
Depth of invasion & $25 / 42(59.52)$ \\
$\leq 1 / 2$ myometrium & $12 / 42(28.57)$ \\
$>1 / 2$ myometrium & $5 / 42(11.90)$ \\
Serosa & \\
LVSI & $8 / 42(19.05)$ \\
Yes & $21 / 42(50.00)$ \\
No & $13 / 42(30.95)$ \\
\hline
\end{tabular}

ESS, endometrial stromal sarcoma; LG, low grade; HG, high grade; UUS, undifferentiated uterine sarcoma; LVSI, Iymphvascular space involvement.

Table 3 The expression patterns of the 10 commonly used IHC markers

\begin{tabular}{lcc}
\hline Markers & Positive (n) & Positive rate (\%) \\
\hline CD10 & $39 / 42$ & 92.86 \\
ER & $22 / 34$ & 64.71 \\
PR & $23 / 34$ & 67.65 \\
Vimentin & $30 / 30$ & 100.00 \\
CyclinD1 & $14 / 16$ & 87.50 \\
SMA & $9 / 37$ & 24.32 \\
S-100 & $0 / 11$ & 0 \\
P53 & $6 / 9$ & 66.67 \\
P16 & $4 / 5$ & 80.00 \\
Ki67 & & \\
$<5 \%$ & $2 / 42$ & 4.76 \\
$5-20 \%$ & $22 / 42$ & 52.38 \\
$>20 \%$ & $18 / 42$ & 42.86 \\
\hline
\end{tabular}

IHC, immunohistochemistry; ER, estrogen receptors; PR, progesterone receptors. 

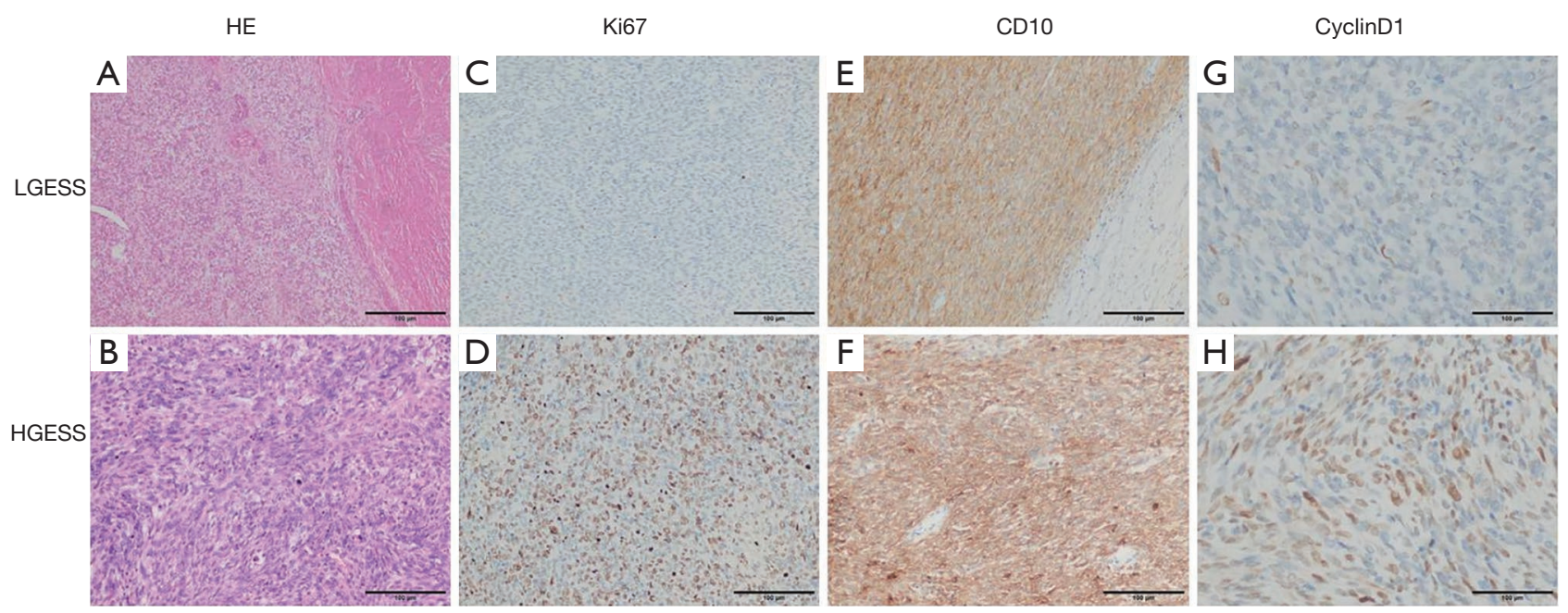

Figure $1 \mathrm{HE}$ staining and IHC contribute to diagnosis of different ESSs. (A,B) HE showing morphologic features in LGESS and HGESS tissues; (C-H) IHC showing expression in LGESS and HGESS. Ki67 index (C and D, ×200), CD10 (E and F, ×200), and CyclinD1 (G and $\mathrm{H}, \times 400)$ respectively. Ki67 and CyclinD1 were strongly expressed in nuclear patterns. CD10 was strongly expressed in cytoplasmic and membranal patterns. HE, hematoxylin and eosin; IHC, immunohistochemistry; LGESS, low-grade ESS; HGESS, high-grade ESS; ESS, endometrial stromal sarcomas.

accounted for the majority (95.24\%) which were showed to have correlation with tumor classification and prognosis.

Hematoxylin and eosin (HE) staining and Ki67/CD10/ CyclinD1 expression by immunohistochemistry (IHC) staining of LGESS and HGESS were shown in Figure 1, and Ki67, CD10, and CyclinD1 were representative indicators usually used in clinic. Tumor cells of LGESS were similar to the endometrial stromal cells in the proliferative stage, without obvious atypia and mitotic figures, and usually showed irregular "tongue-1ike" growth patterns (Figure 1A). HGESS had infiltrative growth, karyotype irregularity with more mitotic figures, and often accompanied by necrosis (Figure 1B). HGESS usually has higher Ki67 index than LGESS (Figure 1C,D). LGESS and HGESS showed positivity for CD10 (Figure 1E,F). CyclinD1 expressed positively in LGESS (Figure $1 G$ ) HGESS (Figure 1H).

\section{Follow-up \& recurrences}

Recurrence after operation developed in 9 (21.43\%) of the 42 patients, with locations of recurrence as follows: pelvic recurrence $(6 / 9,66.67 \%)$, extra-pelvic recurrence $(1 / 9,11.11 \%)$ and both pelvic and extra-pelvic recurrences $(2 / 9,22.22 \%)$ within half a year. Due to a short follow-up time, patient age, menopausal status, tumor size, surgical operation, and lymphatic vascular space invasion did not show any significant association with the recurrence rate. But depth of invasion, tumor classification, and tumor stage all had close correlation to recurrence $(\mathrm{P}<0.05, \mathrm{P}<0.01$, $\mathrm{P}<0.01$ respectively) as shown in Table 4 .

\section{Correlation analysis of related molecular expression}

Of 42 cases, additional immunohistochemical assays had been performed to detect the expression of common molecular markers, including CD10, ER, PR, CyclinD1, and further analyzed the relationship between positive expression and some clinicopathological features as shown in Tables 5,6. CyclinD1 expression closely related to tumor relapse $(\mathrm{P}<0.01)$, and $\mathrm{ER}, \mathrm{PR}$ positivity to tumor classification $(\mathrm{P}<0.05, \mathrm{P}<0.01)$.

\section{Discussion}

ESS, a rare malignant tumor, usually affects the middle-aged women and uterine bleeding is the most common symptom which accounts for $40.48 \%$ in this study. Studies have found that recurrence does exist in patients with retained ovaries (9), and recurrence following a hysterectomy may in part be caused by estrogen stimulation originating from the retained ovaries (6). Our results demonstrated that high rate 
Table 4 Correlations between recurrences and clinicopathological features

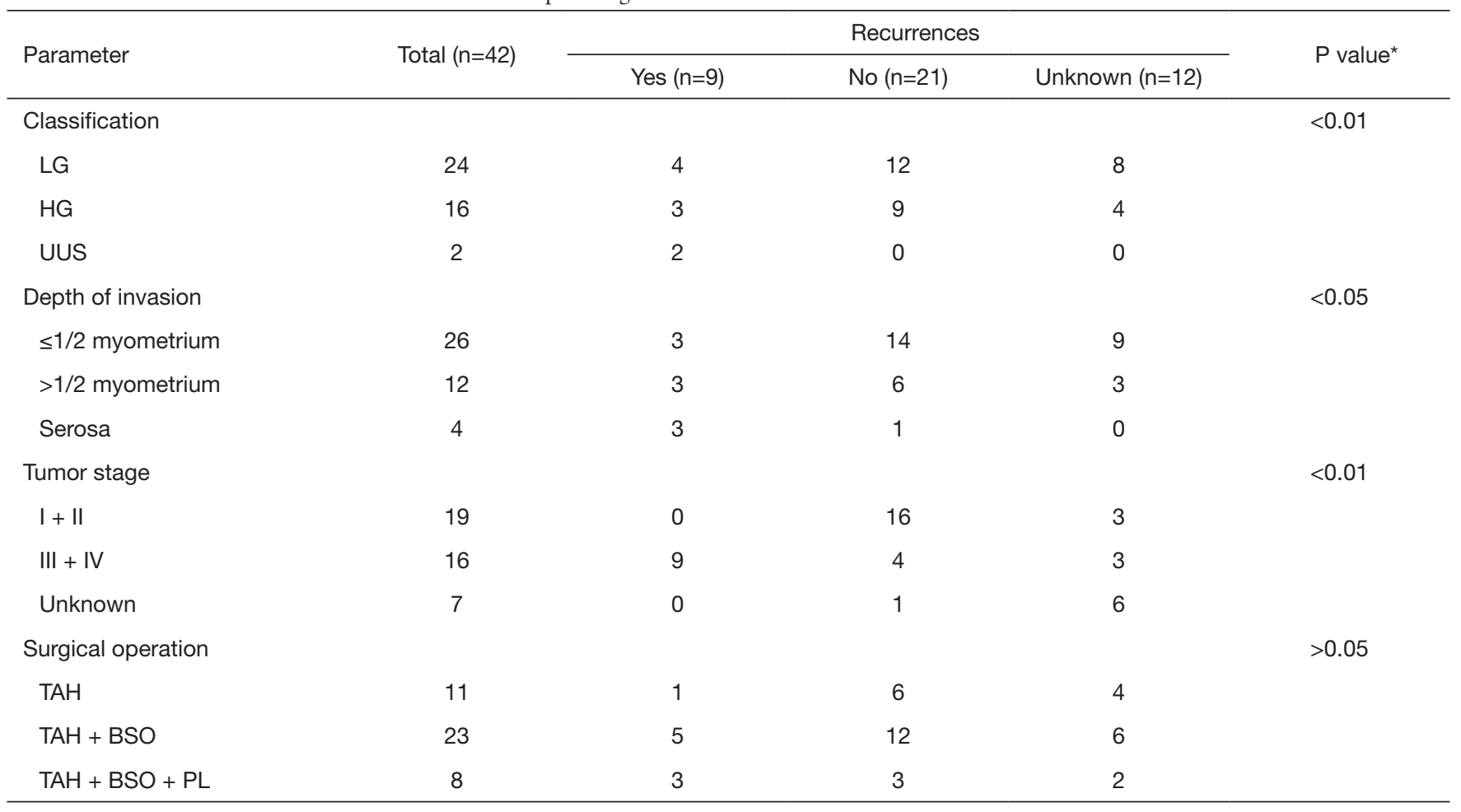

*, two-side Fisher's exact test. LG, low grade; HG, high grade; UUS, undifferentiated uterine sarcoma; TAH, total abdominal hysterectomy; BSO, bilateral salpingo-oophorectomy; PL, pelvic lymphadenectomy.

Table 5 Correlations between CD10/CyclinD1 expression and clinicopathologic features

\begin{tabular}{|c|c|c|c|c|c|c|c|c|}
\hline Parameter & \multicolumn{4}{|c|}{ CD10 } & \multicolumn{4}{|c|}{ CyclinD1 } \\
\hline Classification & & & & $>0.05$ & & & & $>0.05$ \\
\hline$H G$ & 16 & 14 & 2 & & 7 & 7 & 0 & \\
\hline UUS & 2 & 2 & 0 & & 1 & 1 & 0 & \\
\hline$\leq 1 / 2$ myometrium & 26 & 24 & 2 & & 7 & 6 & 1 & $>0.05$ \\
\hline$>1 / 2$ myometrium & 12 & 11 & 1 & & 6 & 5 & 1 & \\
\hline Serosa & 4 & 4 & 0 & & 3 & 3 & 0 & \\
\hline Tumor stage & & & & $>0.05$ & & & & $>0.05$ \\
\hline Relapse & & & & $>0.05$ & & & & $<0.01$ \\
\hline Yes & 9 & 9 & 0 & & 6 & 6 & 0 & \\
\hline No & 21 & 20 & 1 & & 8 & 8 & 0 & \\
\hline Unknown & 12 & 10 & 2 & & 2 & 0 & 2 & \\
\hline
\end{tabular}

*, two-side Fisher's exact test. LG, low grade; HG, high grade; UUS, undifferentiated uterine sarcoma. 
Table 6 Correlations between ER/PR expression and clinicopathologic features.

\begin{tabular}{|c|c|c|c|c|c|c|c|}
\hline Parameter & Total $(n=34)$ & \multicolumn{3}{|c|}{ ER } & \multicolumn{3}{|c|}{ PR } \\
\hline Classification & & & & $<0.05$ & & & $<0.01$ \\
\hline LG & 21 & 17 & 4 & & 18 & 3 & \\
\hline$H G$ & 11 & 5 & 6 & & 5 & 6 & \\
\hline UUS & 2 & 0 & 2 & & 0 & 2 & \\
\hline$\leq 1 / 2$ myometrium & 22 & 15 & 7 & & 15 & 7 & \\
\hline$>1 / 2$ myometrium & 10 & 6 & 4 & & 7 & 3 & \\
\hline Serosa & 2 & 1 & 1 & & 1 & 1 & \\
\hline Tumor stage & & & & $>0.05$ & & & $>0.05$ \\
\hline Relapse & & & & $>0.05$ & & & $>0.05$ \\
\hline Yes & 8 & 3 & 5 & & 4 & 4 & \\
\hline No & 17 & 11 & 6 & & 11 & 6 & \\
\hline Unknown & 9 & 8 & 1 & & 8 & 1 & \\
\hline
\end{tabular}

*, two-side Fisher's exact test. LG, low grade; HG, high grade; UUS, undifferentiated uterine sarcoma; ER, estrogen receptors; PR, progesterone receptors.

of recurrence most affected the pelvic sites (6/9, 66.67\%) within half a year for the vagina and retained ovary, and combination TAH, BSO and PL was not so effective in delaying recurrences.

Only a few patients received long-time follow-up in our research, so it was difficult to get valid statistics data that could evaluate the survival benefits. We found 29 patients were alive with or without evidence of disease, and 9 of them developed recurrence after surgery in a short-term follow-up visit. A study reported overall disease-specific 5- and 10-year survival rates of LGESS were $80-90 \%$ and $70 \%$, respectively (10), so LGESS usually has indolent behavior and a favorable prognosis. He et al. also advocated the integration of PL and BSO into a standardized operation (6), for patients underwent this operation presented a longer disease-free survival in ESSs. Although the standard surgery is still controversial, TAH and BSO was recommended for a patient without requirement to give birth or at menopause as guided by ESMO (European Sarcoma Network Working Group) in 2012 (11). So LGESSs are also treated by TAH and BSO operation which is the major surgical procedure, we also found 5 cases undergoing TAH and BSO developed recurrence until now, however there was no correlation between recurrence and surgical approach in this study. In another words, it is vital for ESSs with early stage to be evaluated for the significance and need for bilateral salpingo-oophorectomy and lymphadenectomy.

IHC often plays an important role in the differential diagnosis of uterine mesenchymal diseases (8). They usually employ IHC markers including ER, PR, Desmin, SMA, h-caldesmon, and CD10, and find that ESSs are predominantly positive for CD10, ER, and PR and negative for desmin, SMA and S-100 (12), which is consistent with our study. Two other key markers we confirmed were Vimentin and CyclinD1 that were highly positive up to $100 \%$ and $87.50 \%$ respectively in this study. When evaluating high-grade uterine sarcomas, CyclinD1 can be included in the immunohistochemical panel as an indicator of YWHAE-FAM22 ESS $(13,14)$. Therefore, CyclinD1 was the most sensitive marker for high-grade endometrial sarcomas with $Y W H A E$ rearrangement (15). However, no genetic rearrangement was further performed in our research for some reasons, we only found $87.50 \%(14 / 16)$ 
ESSs demonstrated CyclinD1 positivity by IHC staining but couldn't differentiate low- and high-grade cases $(\mathrm{P}>0.05)$. Furthermore, we found that there was close relation between CyclinD1 expression and tumor recurrence, but too few samples couldn't prove anything. We should perform gene rearrangement or employ more cases in the future to confirm the relationship between CyclinD1 staining and the relapse. It's necessary to distinguish LGESS from HGESS for their different prognosis and treatment, so the cytogenetic abnormality is essential for pathogenesis of HGESS and responsible for activation of CyclinD1.

CD10, a common acute lymphoblastic lymphoma antigen (CALLA), is frequently expressed in different gyneco-pathological as well as other lesions, which has been reported as a marker for normal and neoplastic endometrial stromal cells previously $(16,17)$, and is nonspecific without relevance to classification of ESSs. Morphological features, extensive sampling and clinic features are more important for correct diagnosis. CD10-negative expression may contribute to the malignant characteristics and recurrence associated with ESSs (6). However, we demonstrated that CD10 was strongly positive in $92.86 \%$ cases, but showed no relation to tumor relapse, tumor classification, depth of invasion and tumor stage. Developments in recent years have shown that IHC can be useful, not only in the differential diagnosis, but also in the prognosis of tumors of female genital tract, and CD10 (27/36) and vimentin were expressed more frequently (6) according to He's report, which was similar to our study.

Estrogen receptors (ER) and progesterone receptors (PR) could contribute to tumor classification and malignancy as shown in our study. Reich et al. reported that ER and PR should be routinely quantified in LGSSs by quantitative immunohistochemical study of ER/PR expression methods (18), and some reports have shown that uterine and extrauterine LGESSs can express ER and PR and sensitive to hormone therapy $(19,20)$. ER, PR $(18)$, and aromatase are usually expressed in ESS, and less commonly in UUS (21-23). In the present study we analyzed the protein expression of ER, PR, CD10, and CyclinD1 in 42 cases with ESSs to correlate the immunohistochemical expression with clinical features, and received similar results which were suggested in previous studies.

Based on these data, we found that $\mathrm{CD} 10^{+} / \mathrm{Vim}^{+} / \mathrm{ER}^{+} /$ $\mathrm{PR}^{+} / \mathrm{CyclinD} 1^{+}$combined with desmin $/ \mathrm{S}-100^{-}$might improve the diagnostic accuracy and differentiation of ESSs. But IHC markers in the diagnosis of ESSs deserve further investigation as a common sense. We believe that application of advanced technologies such as conventional cytogenetics, whole-transcriptome sequencing analysis and fluorescence in situ hybridization (FISH) analysis is needed for further diagnosis. Therefore, further studies are needed to determine clinic treatment and evaluate prognosis through randomized clinical trials and a long-time followup visit.

\section{Acknowledgments}

Funding: This work was supported by the Research Projects of Science and Technology College of Hubei University for Nationalities (NO. KY201723).

\section{Footnote}

Conflicts of Interest: All authors have completed the ICMJE uniform disclosure form (available at http://dx.doi. org/10.21037/tcr.2018.06.12). The authors have no conflicts of interest to declare.

Ethical Statement: The authors are accountable for all aspects of the work in ensuring that questions related to the accuracy or integrity of any part of the work are appropriately investigated and resolved. The study was conducted in accordance with the Declaration of Helsinki (as revised in 2013). The Institutional Research Ethics Committee approved the experimental protocols, the number of ethics approval was 20184301 . The written informed consent was waived due to the retrospective nature of the study.

Open Access Statement: This is an Open Access article distributed in accordance with the Creative Commons Attribution-NonCommercial-NoDerivs 4.0 International License (CC BY-NC-ND 4.0), which permits the noncommercial replication and distribution of the article with the strict proviso that no changes or edits are made and the original work is properly cited (including links to both the formal publication through the relevant DOI and the license). See: https://creativecommons.org/licenses/by-nc-nd/4.0/.

\section{References}

1. Kurman RJ, Carcangiu ML, Herrington CS, et al. WHO Classification of Tumors of Female Reproductive Organs. 4th edition. Lyon, France: IARC Press, 2014.

2. Oliva E, Clement PB, Young RH. Endometrial stromal 
tumors: an update on a group of tumors with a protean phenotype. Adv Anat Pathol 2000;7:257-81.

3. Alessandrini L, Sopracordevole F, Bertola G, et al. Primary extragenital endometrial stromal sarcoma of the lung: first reported case and review of literature. Diagn Pathol 2017;12:36.

4. Haberal A, Kayikçioğlu F, Boran N, et al. Endometrial stromal sarcoma of the uterus: analysis of 25 patients. Eur J Obstet Gynecol Reprod Biol 2003;109:209-13.

5. Leath CA 3rd, Huh WK, Hyde J Jr, et al. A multiinstitutional review of outcomes of endometrial stromal sarcoma. Gynecol Oncol 2007;105:630-4.

6. He L, Li JD, Xiong Y, et al. Clinicopathological and molecular markers associated with prognosis and treatment effectiveness of endometrial stromal sarcoma: a retrospective study in China. Arch Gynecol Obstet 2014;289:383-91.

7. Ali RH, Rouzbahman M. Endometrial stromal tumours revisited: an update based on the 2014 WHO classification. J Clin Pathol 2015;68:325-32.

8. Hwang H, Matsuo K, Duncan K, et al. Immunohistochemical panel to differentiate endometrial stromal sarcoma, uterine leiomyosarcoma and leiomyoma: something old and something new. J Clin Pathol 2015;68:710-7.

9. Beck TL, Singhal PK, Ehrenberg HM, et al. Endometrial stromal sarcoma: analysis of recurrence following adjuvant treatment. Gynecol Oncol 2012;125:141-4.

10. Barney B, Tward JD, Skidmore T, et al. Does radiotherapy or lymphadenectomy improve survival in endometrial stromal sarcoma? Int J Gynecol Cancer 2009;19:1232-8.

11. ESMO/European Sarcoma Network Working Group. Soft tissue and visceral sarcomas: ESMO Clinical Practice Guidelines for diagnosis, treatment and follow-up. Ann Oncol 2014;25 Suppl 3:iii102-12.

12. Mittal K, Soslow R, McCluggage WG. Application of immunohistochemistry to gynecologic pathology. Arch Pathol Lab Med 2008;132:402-23.

13. Kurihara S, Oda Y, Ohishi Y, et al. Coincident expression of beta-catenin and cyclin D1 in endometrial stromal

Cite this article as: Wang F, Yi Y, Luo Y, Chen J. Clinicopathologic correlation of endometrial stromal sarcomas: a retrospective study of 42 cases. Transl Cancer Res 2018;7(4):871-878. doi: 10.21037/tcr.2018.06.12 tumors and related high-grade sarcomas. Mod pathol 2010;23:225-34.

14. Lee CH, Ali RH, Rouzbahman M, et al. Cyclin D1 as a diagnostic immunomarker for endometrial stromal sarcoma with YWHAE-FAM22 rearrangement. Am J Surg Pathol 2012;36:1562-70.

15. Croce S, Hostein I, Ribeiro A, et al. YWHAE rearrangement identified by FISH and RT-PCR in endometrial stromal sarcomas: genetic and pathological correlations. Mod Pathol 2013;26:1390-400.

16. McCluggage WG, Sumathi VP, Maxwell P. CD10 is a sensitive and diagnostically useful immunohistochemical marker of normal endometrial stroma and of endometrial stromal neoplasms. Histopathology 2001;39:273-8.

17. Toki T, Shimizu M, Takagi Y, et al. CD10 is a marker for normal and neoplastic endometrial stromal cells. Int J Gynecol Pathol 2002;21:41-7.

18. Reich O, Regauer S, Urdl W, et al. Expression of oestrogen and progesterone receptors in lowgrade endometrial stromal sarcomas. Br J Cancer 2000;82:1030-4.

19. Fukunaga M, Ishihara A, Ushigome S. Extrauterine lowgrade endometrial stromal sarcoma: report of three cases. Pathol Int 1998;48:297-302.

20. Scribner DR Jr, Walker JL. Low-grade endometrial stromal sarcoma preoperative treatment with DepoLupron and Megace. Gynecol oncol 1998;71:458-60.

21. Ioffe YJ, Li AJ, Walsh CS, et al. Hormone receptor expression in uterine sarcomas: prognostic and therapeutic roles. Gynecol oncol 2009:115:466-71.

22. D'Angelo E, Spagnoli LG, Prat J. Comparative clinicopathologic and immunohistochemical analysis of uterine sarcomas diagnosed using the World Health Organization classification system. Hum pathol 2009;40:1571-85.

23. Kurihara S, Oda Y, Ohishi Y, et al. Endometrial stromal sarcomas and related high-grade sarcomas: immunohistochemical and molecular genetic study of 31 cases. Am J Surg Pathol 2008;32:1228-38. 